\title{
Denseness and Borel complexity of some sets of vector measures
}

\author{
by \\ ZBIGNIEW LiPECKI (Wrocław) \\ Dedicated to Lech Drewnowski on his 60th birthday
}

\begin{abstract}
Let $\nu$ be a positive measure on a $\sigma$-algebra $\Sigma$ of subsets of some set and let $X$ be a Banach space. Denote by ca $(\Sigma, X)$ the Banach space of $X$-valued measures on $\Sigma$, equipped with the uniform norm, and by ca $(\Sigma, \nu, X)$ its closed subspace consisting of those measures which vanish at every $\nu$-null set. We are concerned with the subsets $\mathcal{E}_{\nu}(X)$ and $\mathcal{A}_{\nu}(X)$ of $\operatorname{ca}(\Sigma, X)$ defined by the conditions $|\varphi|=\nu$ and $|\varphi| \geq \nu$, respectively, where $|\varphi|$ stands for the variation of $\varphi \in \mathrm{ca}(\Sigma, X)$. We establish necessary and sufficient conditions that $\mathcal{E}_{\nu}(X)$ [resp., $\left.\mathcal{A}_{\nu}(X)\right]$ be dense in ca $(\Sigma, \nu, X)$ [resp., ca $\left.(\Sigma, X)\right]$. We also show that $\mathcal{E}_{\nu}(X)$ and $\mathcal{A}_{\nu}(X)$ are always $G_{\delta^{-}}$sets and establish necessary and sufficient conditions that they be $F_{\sigma}$-sets in the respective spaces.
\end{abstract}

1. Introduction. Let $\nu$ be a positive measure on a $\sigma$-algebra $\Sigma$ of subsets of some set. In [8] the author was concerned with the question of when we can find a Banach space $X$ and a ( $\sigma$-additive vector) measure $\varphi: \Sigma \rightarrow X$ whose variation $|\varphi|$ equals $\nu$. In particular, it was proved that this is the case when there exists a nonatomic positive finite measure $\lambda$ on $\Sigma$ equivalent to $\nu$, i.e., for every $E \in \Sigma$ we have $\nu(E)=0$ if and only if $\lambda(E)=0$. Moreover, we can then take for $X$ an arbitrary infinitedimensional Banach space and choose $\varphi$ with relatively compact range ([8, Theorem 1]). This is an extension of previous results due to Thomas [11], Janicka and Kalton [5], and Drewnowski and the author [3].

In this paper we study some topological properties of the set $\mathcal{E}_{\nu}(X)$ of those measures $\varphi$ on $\Sigma$ with values in a fixed Banach space $X$ for which $|\varphi|=\nu$. In the special case where $\nu=\infty \cdot \lambda$ for some nonatomic positive

2000 Mathematics Subject Classification: Primary 28B05, 46G10, 46E27, 54H05; Secondary $46 \mathrm{~A} 16$.

Key words and phrases: positive measure, Banach space, vector measure, variation, relatively compact range, Banach space of vector measures, dense set, closed set, $G_{\delta}$-set, $F_{\sigma}$-set.

The main results of this paper were presented at the 30th Winter School on Abstract Analysis (Lhota nad Rohanovem, Czech Republic, 2002). 
finite measure $\lambda$ on $\Sigma$ and $X$ is infinite-dimensional, it was shown in [3] that $\mathcal{E}_{\nu}(X)$ is a dense $G_{\delta}$-set in the Banach space ca $(\Sigma, \nu, X)$ of those measures $\varphi: \Sigma \rightarrow X$ which vanish at every $\nu$-null set, equipped with the uniform norm. (A closely related result was previously established by Anantharaman and Garg [1].) We prove that the denseness assertion holds essentially only in that case while the other assertion remains valid for arbitrary $\nu$ and $X$ (Theorem 2 of Section 3 and Theorem 4(a) of Section 4, respectively). We also show that $\mathcal{E}_{\nu}(X)$ is an $F_{\sigma}$-set in ca $(\Sigma, \nu, X)$ if and only if $\mathcal{E}_{\nu}(X)$ is empty or $\nu$ is atomic or $X$ is finite-dimensional. In each of those cases, $\mathcal{E}_{\nu}(X)$ is actually closed (Theorem 5 of Section 5 ).

We also make a parallel study of the set $\mathcal{A}_{\nu}(X)$ of those measures $\varphi: \Sigma \rightarrow X$ for which $|\varphi| \geq \nu$. For example we show that $\mathcal{A}_{\nu}(X)$ is dense in the Banach space ca $(\Sigma, X)$ of all measures $\varphi: \Sigma \rightarrow X$, equipped with the uniform norm, if and only if either $\nu=0$, or there exists a nonatomic positive finite measure $\lambda$ on $\Sigma$ equivalent to $\nu$ and $X$ is infinite-dimensional (Theorem 3 of Section 3 ). In fact, some of our results on $\mathcal{A}_{\nu}(X)$ are simple consequences of the corresponding results on $\mathcal{E}_{\nu}(X)$ while for other results on $\mathcal{A}_{\nu}(X)$ the converse holds.

Both results and methods of [3] play an essential role in this paper. We note that [3] owes, in turn, some ideas to [1] and [5]. We also make use of some results of [8] and of more or less standard material on vector measures, including a theorem of Bartle, Dunford and Schwartz, and a version of the Lebesgue decomposition theorem.

Most of the notation we need and some auxiliary results are presented in Section 2. Some more notation is introduced in Section 5 (see the passage following Lemma 8) and in the final Section 6. Sections 3, 4 and 5 deal with denseness, $G_{\delta}$-sets and $F_{\sigma}$-sets, respectively. Section 4 is independent of Section 3 as far as the proofs are concerned. Section 6 discusses briefly counterparts of our main results for vector measures with relatively compact range.

2. Notation, terminology and auxiliary results. Throughout the paper $S$ stands for a (nonempty) set and $\Sigma$ for a $\sigma$-algebra of subsets of $S$. Moreover, $\nu$ stands for a positive measure on $\Sigma$, i.e., a $\sigma$-additive function on $\Sigma$ with values in $[0, \infty]$ such that $\nu(\emptyset)=0$.

We say that $A \in \Sigma$ is a $\nu$-atom if $\nu(A)>0$ and for every $E \in \Sigma$ with $A \supset E \in \Sigma$ we have $\nu(E)=0$ or $\nu(A \backslash E)=0$. The measure $\nu$ is called atomic if every $E \in \Sigma$ with $\nu(E)>0$ contains a $\nu$-atom. The measure $\nu$ is called nonatomic (or atomless) if it has no atom. Given $T \in \Sigma$, we set

$$
\nu_{T}(E)=\nu(E \cap T) \quad \text { for all } E \in \Sigma .
$$

Clearly, $\nu_{T}$ is again a positive measure on $\Sigma$. 
For positive measures $\nu_{1}$ and $\nu_{2}$ on $\Sigma$ we write $\nu_{1} \ll \nu_{2}$ [resp., $\nu_{1} \equiv \nu_{2}$ ] provided that for every $E \in \Sigma$ we have $\nu_{1}(E)=0$ if [resp., if and only if] $\nu_{2}(E)=0$. If the former condition holds, $\nu_{1}$ is $\nu_{2}$-saturated in the terminology of $\left[4\right.$, p. 169]. If the latter condition holds, $\nu_{1}$ and $\nu_{2}$ are said to be equivalent.

Throughout the paper $X$ stands for a (real or complex) Banach space, with the norm denoted by $\|\cdot\|$. Let $\varphi: \Sigma \rightarrow X$ be a ( $\sigma$-additive vector) measure. For $E \in \Sigma$ we define $|\varphi|(E)$ as the supremum of the sums $\sum_{i=1}^{\infty}\left\|\varphi\left(E_{i}\right)\right\|$, where $\left\{E_{1}, E_{2}, \ldots\right\}$ is a $\Sigma$-partition of $E$. The set function $|\varphi|$ is a positive measure on $\Sigma$, called the variation of $\varphi$. Given $T \in \Sigma$, we set

$$
\varphi_{T}(E)=\varphi(E \cap T) \quad \text { for all } E \in \Sigma .
$$

Clearly, $\varphi_{T}$ is again a measure on $\Sigma$.

We say that $A \in \Sigma$ is a $\varphi$-atom if $A$ is a $|\varphi|$-atom. As is easily seen, $A \in \Sigma$ is a $\varphi$-atom if and only if $\varphi(A) \neq 0$ and for every $E \in \Sigma$ with $A \supset E \in \Sigma$ we have $\varphi_{E}=0$ or $\varphi_{A \backslash E}=0$. The measure $\varphi$ is called atomic if $|\varphi|$ is atomic (equivalently, every $E \in \Sigma$ with $\varphi(E) \neq 0$ contains a $\varphi$-atom). The measure $\varphi$ is called nonatomic (or atomless) if it has no atom.

We write $\varphi \ll \nu[$ resp., $\varphi \equiv \nu]$ provided that $|\varphi| \ll \nu[$ resp., $|\varphi| \equiv \nu]$. We write $\varphi \perp \nu$ provided that there exists $T \in \Sigma$ with $|\varphi|(T)=0$ and $\nu(S \backslash T)=0$.

We denote by $\mathrm{ca}(\Sigma, X)$ the Banach space of all measures $\varphi: \Sigma \rightarrow X$ equipped with the uniform norm

$$
\|\varphi\|=\sup \{\|\varphi(E)\|: E \in \Sigma\}
$$

(see $[2$, pp. 29-30]). The notation ca $(\Sigma, \mathbb{R})$ is abbreviated to ca $(\Sigma)$. Thus, $\mathrm{ca}_{+}(\Sigma)$ stands for the set of all positive finite measures on $\Sigma$.

We set

$$
\operatorname{ca}(\Sigma, \nu, X)=\{\varphi \in \operatorname{ca}(\Sigma, X): \varphi \ll \nu\} .
$$

This is a closed (linear) subspace of $\mathrm{ca}(\Sigma, X)$. Moreover, we set

$$
\operatorname{cca}(\Sigma, X)=\{\varphi \in \operatorname{ca}(\Sigma, X): \varphi(\Sigma) \text { is relatively compact }\} \text {. }
$$

This is also a closed subspace of $\operatorname{ca}(\Sigma, X)$. It appears below only in Theorem 1 and then in Section 6.

For each $\nu$ we define three sets of vector measures as follows:

$$
\begin{aligned}
& \mathcal{E}_{\nu}(X)=\{\varphi \in \operatorname{ca}(\Sigma, X):|\varphi|=\nu\}, \\
& \mathcal{A}_{\nu}(X)=\{\varphi \in \operatorname{ca}(\Sigma, X):|\varphi| \geq \nu\}, \\
& \mathcal{B}_{\nu}(X)=\{\varphi \in \operatorname{ca}(\Sigma, X):|\varphi| \leq \nu\} .
\end{aligned}
$$

The sets $\mathcal{E}_{\nu}(X)$ and $\mathcal{A}_{\nu}(X)$ do not seem to have appeared explicitly in the literature before, while $\mathcal{B}_{\nu}(X)$ appears, in another context, in [10, p. $682 \mathrm{ff}$.]. (Our notation differs from that of [10].) We shall be mainly concerned with $\mathcal{E}_{\nu}(X)$ and $\mathcal{A}_{\nu}(X)$ in what follows, the role of $\mathcal{B}_{\nu}(X)$ being auxiliary only. 
Clearly, we have

$$
\mathcal{E}_{\nu}(X)=\mathcal{A}_{\nu}(X) \cap \mathcal{B}_{\nu}(X) .
$$

We shall tacitly use this formula on several occasions, combined with the following simple lemma.

Lemma 1. The set $\mathcal{B}_{\nu}(X)$ is closed in $\mathrm{ca}(\Sigma, X)$.

Proof. We have

$$
\begin{aligned}
\mathcal{B}_{\nu}(X) & =\{\varphi \in \operatorname{ca}(\Sigma, X):\|\varphi(E)\| \leq \nu(E) \text { for all } E \in \Sigma\} \\
& =\bigcap_{E \in \Sigma}\{\varphi \in \operatorname{ca}(\Sigma, X):\|\varphi(E)\| \leq \nu(E)\} .
\end{aligned}
$$

Thus, $\mathcal{B}_{\nu}(X)$ is even closed in the topology of pointwise convergence in ca $(\Sigma, X)$.

The next lemma will be needed in the proof of Theorem 2 .

Lemma 2. If $\nu_{F}$ is nonatomic for some $F \in \Sigma$ with $0<\nu(F)<\infty$ and $X$ is infinite-dimensional, then $\mathcal{B}_{\nu}(X)$ is nowhere dense in $\operatorname{ca}(\Sigma, \nu, X)$.

Proof. In view of Lemma 1 , it is enough to show that $\mathcal{B}_{\nu}(X)$ has empty interior in $\mathrm{ca}(\Sigma, \nu, X)$. To this end, fix $\varphi \in \mathcal{B}_{\nu}(X)$ and $\varepsilon>0$. By [3, Proposition 2.2], there exists $\psi_{0} \in \operatorname{ca}\left(\Sigma, \nu_{F}, X\right)$ with

$$
\left\|\psi_{0}\right\|<\varepsilon \quad \text { and }\left|\psi_{0}\right|(F)>\nu(F)+|\varphi|(F) .
$$

Set $\psi=\varphi+\psi_{0}$. Clearly, $\psi \in \operatorname{ca}(\Sigma, \nu, X)$ and $\|\varphi-\psi\|<\varepsilon$. Moreover, $|\psi|(F)>\nu(F)$, and so $\psi \notin \mathcal{B}_{\nu}(X)$. Thus, $\varphi$ is not an interior point of $\mathcal{B}_{\nu}(X)$ in $\mathrm{ca}(\Sigma, \nu, X)$.

We shall frequently apply the following version of a fundamental theorem due to R. G. Bartle, N. Dunford and J. T. Schwartz (see [2, Corollary I.2.6]). (BDS) For every $\varphi \in \mathrm{ca}(\Sigma, X)$ there exists $\lambda \in \mathrm{ca}_{+}(\Sigma)$ with $\varphi \equiv \lambda$.

The next lemma will be used in the proofs of Lemma 4(c) and Theorems 1 and 3 .

Lemma 3. Let $\varphi \in \mathcal{A}_{\nu}(X)$. Then

(a) There exists $T \in \Sigma$ with $\varphi_{T} \equiv \nu$.

(b) There exists $\lambda \in \mathrm{ca}_{+}(\Sigma)$ with $\nu \equiv \lambda$.

Proof. According to (BDS), there exists $\lambda_{0} \in \operatorname{ca}_{+}(\Sigma)$ with $\varphi \equiv \lambda_{0}$. Hence $\nu \ll \lambda_{0}$. Set

$$
\Sigma_{0}=\left\{E \in \Sigma: \nu(E)=0 \text { and } \lambda_{0}(E)>0\right\} .
$$

If $\Sigma_{0}$ is empty, put $T=S$. Otherwise, let

$$
\alpha=\sup \left\{\lambda_{0}(E): E \in \Sigma_{0}\right\},
$$


choose $E_{n} \in \Sigma_{0}$ with $\lambda_{0}\left(E_{n}\right) \rightarrow \alpha$, and put

$$
T=S \backslash \bigcup_{n=1}^{\infty} E_{n} .
$$

As is easily seen, (b) holds with $\lambda=\left(\lambda_{0}\right)_{T}$. This also yields (a). (Note that (b) also follows from (BDS) and $[4,(3.16 .3)$ and (3.16.4)].)

In the proofs of Lemma 4(c) and Theorem 3 we shall apply some consequences of the following version of the Lebesgue decomposition theorem for vector measures. Given $\lambda \in \mathrm{ca}_{+}(\Sigma)$ and $\varphi \in \mathrm{ca}(\Sigma, X)$, there exists a unique $\psi \in \operatorname{ca}(\Sigma, X)$ with

$$
\psi \ll \lambda \text { and } \varphi-\psi \perp \lambda .
$$

See, e.g., [9, Theorem 9] or [6, Corollary 2] for more general results, but note that the relation $\ll$ is defined wrongly in [9]. In fact, we have $\psi=\varphi_{T}$ for some $T \in \Sigma$, and the present version also follows from the classical Lebesgue decomposition theorem and (BDS).

Set

$$
\mathcal{A}_{\nu}(\lambda, X)=\mathcal{A}_{\nu}(X) \cap \mathrm{ca}(\Sigma, \lambda, X) .
$$

(In the special case where $\nu=r \lambda$ with $r>0$, the set $\mathcal{A}_{\nu}(\lambda, X)$ appears in $[3$, pp. 23-24].) With this notation, we have

(LP) The operator $P_{\lambda}: \operatorname{ca}(\Sigma, X) \rightarrow \operatorname{ca}(\Sigma, X)$ defined by $P_{\lambda}(\varphi)=\psi$, where $\psi$ satisfies (1), is a continuous linear projection with range $\mathrm{ca}(\Sigma, \lambda, X)$. Moreover, if $\nu \ll \lambda$, then

$$
P_{\lambda}^{-1}\left(\mathcal{A}_{\nu}(\lambda, X)\right)=\mathcal{A}_{\nu}(X)
$$

In particular, $\mathcal{A}_{\nu}(\lambda, X)$ is dense in ca $(\Sigma, \lambda, X)$ if and only if $\mathcal{A}_{\nu}(X)$ is dense in $\mathrm{ca}(\Sigma, X)$.

The first assertion is straightforward. As to the second, it is enough to note that for every $\varphi \in \operatorname{ca}(\Sigma, X)$ we have

$$
|\varphi|=\left|P_{\lambda}(\varphi)+\left(\varphi-P_{\lambda}(\varphi)\right)\right|=\left|P_{\lambda}(\varphi)\right|+\left|\varphi-P_{\lambda}(\varphi)\right|
$$

and $\left|\varphi-P_{\lambda}(\varphi)\right| \perp \nu$. Hence

$$
|\varphi| \geq \nu \text { if and only if }\left|P_{\lambda}(\varphi)\right| \geq \nu
$$

The last assertion follows from the previous ones.

The next lemma will be used in the proofs of Lemma 5 and Theorems 2, 3 and 5.

LEMMA 4. (a) If $\nu$ has an atom, then $\mathcal{E}_{\nu}(X)$ is nowhere dense in $\operatorname{ca}(\Sigma, \nu, X)$.

(b) If $\nu$ has an atom, then $\mathcal{A}_{\nu}(\nu, X)$ is not dense in $\operatorname{ca}(\Sigma, \nu, X)$.

(c) If $\nu$ is atomic, then $\mathcal{E}_{\nu}(X)$ and $\mathcal{A}_{\nu}(X)$ are closed in $\mathrm{ca}(\Sigma, X)$. 
Proof. To show (a) and (b), fix a $\nu$-atom $A$. For every $\varphi \in \operatorname{ca}(\Sigma, \nu, X)$ we have $|\varphi|(A)=\|\varphi(A)\|$. This yields

$$
\begin{aligned}
& \mathcal{E}_{\nu}(X) \subset\{\varphi \in \operatorname{ca}(\Sigma, \nu, X):\|\varphi(A)\|=\nu(A)\}, \\
& \mathcal{A}_{\nu}(\nu, X) \subset\{\varphi \in \operatorname{ca}(\Sigma, \nu, X):\|\varphi(A)\| \geq \nu(A)\},
\end{aligned}
$$

and (a) and (b) follow.

To prove (c) it is enough, in view of Lemma 1, to establish the closedness of $\mathcal{A}_{\nu}(X)$. Suppose $\nu$ is atomic and nonzero, and $\mathcal{A}_{\nu}(X)$ is nonempty. Choose $\lambda$ according to Lemma $3(\mathrm{~b})$. We shall show that $\mathcal{A}_{\nu}(\lambda, X)$ is closed in $\operatorname{ca}(\Sigma, \lambda, X)$. This yields the assertion, by (LP).

There exists a (countable) family $\left\{A_{i}: i \in I\right\}$ of pairwise disjoint $\lambda$-atoms with $S=\bigcup_{i \in I} A_{i}$. The closedness of $\mathcal{A}_{\nu}(\lambda, X)$ is an obvious consequence of the formula

$$
\mathcal{A}_{\nu}(\lambda, X)=\left\{\varphi \in \operatorname{ca}(\Sigma, \lambda, X):\left\|\varphi\left(A_{i}\right)\right\| \geq \nu\left(A_{i}\right) \text { for all } i \in I\right\} .
$$

Let $\varphi \in \operatorname{ca}(\Sigma, \lambda, X)$. As in the proof of (a) and (b), we get

$$
|\varphi|\left(A_{i}\right)=\left\|\varphi\left(A_{i}\right)\right\| \quad \text { for all } i \in I .
$$

This yields the inclusion " $\subset$ " in (2). To establish the other inclusion, fix $E \in \Sigma$, and observe that

$$
E=\left(\bigcup_{i \in J} A_{i}\right) \triangle F \quad \text { for some } J \subset I \text { and } F \in \Sigma \text { with } \lambda(F)=0 .
$$

It follows that

$$
|\varphi|(E)=|\varphi|\left(\bigcup_{i \in J} A_{i}\right)=\sum_{i \in J}|\varphi|\left(A_{i}\right) .
$$

If $\left\|\varphi\left(A_{i}\right)\right\| \geq \nu\left(A_{i}\right)$ for all $i \in I$, this implies

$$
|\varphi|(E) \geq \sum_{i \in J} \nu\left(A_{i}\right)=\nu(E),
$$

completing the proof.

The following lemma will be used in the proofs of Theorem 2 and Lemma 8.

Lemma 5. If $\nu \neq 0$, then $\mathcal{E}_{\nu}(X)$ has empty interior in $\mathrm{ca}(\Sigma, \nu, X)$.

Proof. In view of Lemma 4(a), we may assume that $\nu$ is nonatomic. Assume, moreover, that $\mathcal{E}_{\nu}(X)$ is nonempty, and fix $\varphi \in \mathcal{E}_{\nu}(X)$ and $\varepsilon>0$. According to (BDS), $\nu \equiv \lambda$ for some $\lambda \in \mathrm{ca}_{+}(\Sigma)$. On account of [2, Theorem I.2.1 or Corollary I.2.6], there exists $\delta>0$ such that $\left\|\varphi_{E}\right\|<\varepsilon$ whenever $E \in \Sigma$ and $\lambda(E)<\delta$. Fix $E_{0} \in \Sigma$ with $0<\lambda\left(E_{0}\right)<\delta$. Set $\psi=\varphi_{S \backslash E_{0}}$. Clearly,

$$
\psi \in \operatorname{ca}(\Sigma, \nu, X) \backslash \mathcal{E}_{\nu}(X) \text { and }\|\varphi-\psi\|<\varepsilon .
$$

It follows that $\varphi$ is not in the interior of $\mathcal{E}_{\nu}(X)$.

The next lemma will be used in the proofs of Theorems 2, 3 and 5 . 
LEMma 6. If $X$ is finite-dimensional, then $\mathcal{E}_{\nu}(X)$ and $\mathcal{A}_{\nu}(X)$ are closed in $\operatorname{ca}(\Sigma, X)$.

Proof. As is well known, there exists a constant $C$ such that

$$
|\varphi|(S) \leq C\|\varphi\| \quad \text { for all } \varphi \in \operatorname{ca}(\Sigma, X) .
$$

(We can take $C=2 \operatorname{dim} X$ or $4 \operatorname{dim} X$ according as $X$ is real or complex, respectively.)

Given $\varphi_{1}, \varphi_{2} \in \operatorname{ca}(\Sigma, X)$, we have

$$
\left|\varphi_{1}\right|-\left|\varphi_{2}\right| \leq\left|\varphi_{1}-\varphi_{2}\right| \text {. }
$$

Combining (3) and (4), we get

$$
\left\|\left|\varphi_{1}\right|-\left|\varphi_{2}\right|\right\| \leq\left|\varphi_{1}-\varphi_{2}\right|(S) \leq C\left\|\varphi_{1}-\varphi_{2}\right\| \text {. }
$$

It follows that the map $M: \operatorname{ca}(\Sigma, X) \rightarrow \operatorname{ca}(\Sigma)$ defined, for $\varphi \in \operatorname{ca}(\Sigma, X)$, by $M(\varphi)=|\varphi|$ is continuous. To complete the proof, it is now enough to observe that

$$
\begin{aligned}
& \mathcal{E}_{\nu}(X)=M^{-1}(\{\mu \in \operatorname{ca}(\Sigma): \mu=\nu\}), \\
& \mathcal{A}_{\nu}(X)=M^{-1}(\{\mu \in \operatorname{ca}(\Sigma): \mu \geq \nu\}) .
\end{aligned}
$$

We proceed to a result which will be used in the proof of Theorem 5 . This result can also be considered a supplement to [8].

TheOREM 1. If $\mathcal{A}_{\nu}(X)$ is nonempty, then so is $\mathcal{E}_{\nu}(X) \cap \operatorname{cca}(\Sigma, X)$.

Proof. Fix $\varphi \in \mathcal{A}_{\nu}(X)$. We first consider two special cases.

CASE 1: $\varphi$ is nonatomic. Then $\nu$ is also nonatomic, by Lemma 3(a). The assertion then follows from Lemma $3(\mathrm{~b})$ and [8, Lemma 4] if $X$ is infinitedimensional. Otherwise, $\nu$ is finite (cf. (3) in the proof of Lemma 6) and the assertion is obvious.

CASE 2: $\varphi$ is atomic. In view of (BDS), we can choose pairwise disjoint $A_{i} \in \Sigma, i=1,2, \ldots$, such that each $A_{i}$ is either a $\varphi$-atom or $A_{i}=\emptyset$, and $S=\bigcup_{i=1}^{\infty} A_{i}$. Clearly, for each $i$, we then have $\nu\left(A_{i}\right)=0$ or $A_{i}$ is a $\nu$-atom. Take $0 \leq t_{i} \leq 1$ with

$$
t_{i}|\varphi|\left(A_{i}\right)=\nu\left(A_{i}\right)
$$

This yields

$$
t_{i}|\varphi|\left(A_{i} \cap E\right)=\nu\left(A_{i} \cap E\right) \quad \text { for all } i \text { and } E \in \Sigma .
$$

Set

$$
\psi(E)=\int_{E} \sum_{i=1}^{\infty} t_{i} 1_{A_{i}} d \varphi \quad \text { for } E \in \Sigma .
$$

(The integral we use here is that of [2, pp. 5-6].) By [2, Theorem II.4.1], we get $\psi \in \operatorname{ca}(\Sigma, X)$. Since

$$
\psi\left(A_{i} \cap E\right)=t_{i} \varphi\left(A_{i} \cap E\right),
$$


we have

$$
|\psi|\left(A_{i} \cap E\right)=t_{i}|\varphi|\left(A_{i} \cap E\right),
$$

which, together with (5), implies

$$
|\psi|\left(A_{i} \cap E\right)=\nu\left(A_{i} \cap E\right) \quad \text { for all } i \text { and } E \in \Sigma .
$$

Hence $|\psi|=\nu$. It follows that $\psi \in \operatorname{cca}(\Sigma, X)$ (see, e.g., [9, Theorem 3]).

In the general case, appealing to (BDS) again, we can take disjoint $S_{1}, S_{2} \in \Sigma$ with $\varphi_{S_{1}}$ nonatomic and $\varphi_{S_{2}}$ atomic and $S=S_{1} \cup S_{2}$. Clearly,

$$
\varphi_{S_{j}} \in \mathcal{A}_{\nu_{S_{j}}}(X) \text { for } j=1,2 \text {. }
$$

By what we have proved so far, there exist

$$
\psi_{j} \in \mathcal{E}_{\nu_{S_{j}}}(X) \cap \operatorname{cca}(\Sigma, X), \quad j=1,2 .
$$

Set $\psi=\psi_{1}+\psi_{2}$. It is plain that $\psi \in \mathcal{E}_{\nu}(X) \cap \operatorname{cca}(\Sigma, X)$.

3. Denseness. Recall that throughout the paper, $\nu$ stands for a positive measure on a $\sigma$-algebra $\Sigma$ of subsets of a set $S$, and $X$ for a Banach space.

We shall now establish two denseness results. The implication (iv) $\Rightarrow(\mathrm{i})$ of the first of them is essentially a special case of [3, Theorem 4.1] (see also [1, Theorem 2.4] for a closely related result).

THEOREM 2. The following four conditions are equivalent:

(i) $\mathcal{E}_{\nu}(X)$ is dense in $\mathrm{ca}(\Sigma, \nu, X)$;

(ii) $\mathcal{E}_{\nu}(X)$ is not nowhere dense in $\mathrm{ca}(\Sigma, \nu, X)$;

(iii) $\mathcal{E}_{\nu}(X)$ is of second category in $\mathrm{ca}(\Sigma, \nu, X)$;

(iv) either $\nu=0$, or $\nu=\infty \cdot \lambda$ for some nonatomic $\lambda \in \mathrm{ca}_{+}(\Sigma)$ and $X$ is infinite-dimensional.

Proof. As mentioned above, (iv) implies (i). Clearly, (i) implies (ii) and (iii) implies (ii). By Theorem 4(a) below and the Baire category theorem, (i) implies (iii). We shall complete the proof by showing that (ii) implies (iv).

Suppose (ii) holds and $\nu \neq 0$. By Lemma 4(a), $\nu$ is then nonatomic. It now follows from Lemmas 5 and 6 that $X$ is infinite-dimensional. Therefore, $\nu(\Sigma)=\{0, \infty\}$, by Lemma 2. Combined with an application of (BDS), this yields (iv).

THEOREM 3. The following three conditions are equivalent:

(i) $\mathcal{A}_{\nu}(X)$ is dense in ca $(\Sigma, X)$;

(ii) $\mathcal{A}_{\nu}(\nu, X)$ is dense in $\mathrm{ca}(\Sigma, \nu, X)$;

(iii) either $\nu=0$, or $\nu \equiv \lambda$ for some nonatomic $\lambda \in \mathrm{ca}_{+}(\Sigma)$ and $X$ is infinite-dimensional.

Proof. In view of Lemma 3(b), the equivalence of (i) and (ii) follows from (LP). We shall show that (ii) and (iii) are also equivalent. 
Suppose (ii) holds and $\nu \neq 0$. Then $0 \notin \mathcal{A}_{\nu}(X)$, and so, in view of Lemma $6, X$ is infinite-dimensional. Moreover, Lemma 4(b) implies that $\nu$ is nonatomic. Thus, (iii) holds, by Lemma 3(b).

Suppose (iii) holds. The case where $\nu=0$ is obvious. The other case follows from Theorem 2 , (iv) $\Rightarrow(\mathrm{i}$ ), since we then have

$$
\mathcal{E}_{\infty \cdot \lambda}(X) \subset \mathcal{A}_{\nu}(\nu, X) .
$$

4. $G_{\delta}$-sets. We start with an auxiliary result which is a variant of $[3$, Proposition 3.3(b)]. The proof below is a modification of the corresponding proof given in [3, pp. 22-23].

Lemma 7. Given $\lambda, \mu \in \mathrm{ca}_{+}(\Sigma)$ and $\delta>0$, the set $\mathcal{F}(\lambda, \mu, \delta)$ of all $\varphi \in \mathrm{ca}(\Sigma, X)$ such that there exists $E \in \Sigma$ with

$$
\lambda(E) \geq \delta \quad \text { and } \quad|\varphi|(F) \leq \mu(F) \quad \text { whenever } F \in \Sigma \text { and } F \subset E
$$

is closed in $\mathrm{ca}(\Sigma, X)$.

Proof. Let $\varphi_{0}$ be in the closure of $\mathcal{F}(\lambda, \mu, \delta)$. Choose $\varphi_{n}$ in $\mathcal{F}(\lambda, \mu, \delta)$ so that $\left\|\varphi_{0}-\varphi_{n}\right\| \leq n^{-2}$ for all $n$. By definition, there exist $E_{n} \in \Sigma$ with

$\lambda\left(E_{n}\right) \geq \delta \quad$ and $\quad\left|\varphi_{n}\right|(F) \leq \mu(F) \quad$ whenever $F \in \Sigma$ and $F \subset E_{n}$. Set $E_{0}=\bigcap_{k=1}^{\infty} \bigcup_{n=k}^{\infty} E_{n}$. Clearly, $\lambda\left(E_{0}\right) \geq \delta$. Fix $F \subset E_{0}$ and $k$. There exist pairwise disjoint $F_{n} \in \Sigma, n \geq k$, with

$$
F_{n} \subset E_{n} \text { and } F=\bigcup_{n=k}^{\infty} F_{n} .
$$

It follows that

$$
\begin{aligned}
\left\|\varphi_{0}(F)\right\| & \leq \sum_{n=k}^{\infty}\left\|\varphi_{0}\left(F_{n}\right)\right\| \leq \sum_{n=k}^{\infty}\left\|\varphi_{0}\left(F_{n}\right)-\varphi_{n}\left(F_{n}\right)\right\|+\sum_{n=k}^{\infty}\left\|\varphi_{n}\left(F_{n}\right)\right\| \\
& \leq \sum_{n=k}^{\infty} n^{-2}+\sum_{n=k}^{\infty} \mu\left(F_{n}\right)=\sum_{n=k}^{\infty} n^{-2}+\mu(F) .
\end{aligned}
$$

Hence $\left\|\varphi_{0}(F)\right\| \leq \mu(F)$. Consequently, $\varphi_{0} \in \mathcal{F}(\lambda, \mu, \delta)$, which completes the proof.

Part (a) of the following result generalizes the corresponding assertion of [3, Theorem 4.1] (see also [1, Corollary 2.4]). Namely, what is considered in [3] is the situation where $\nu=\infty \cdot \lambda$ for some nonatomic probability measure $\lambda$ on $\Sigma$.

TheOREM 4. (a) $\mathcal{E}_{\nu}(X)$ is a $G_{\delta}$-set in $\mathrm{ca}(\Sigma, X)$.

(b) $\mathcal{A}_{\nu}(X)$ is a $G_{\delta}$-set in $\mathrm{ca}(\Sigma, X)$.

Proof. Part (a) follows from (b) and Lemma 1. We first establish (b) under the additional assumption that $\nu$ be finite. With the notation of 
Lemma 7, we then have

$$
\mathrm{ca}(\Sigma, X) \backslash \mathcal{A}_{\nu}(X)=\bigcup_{n, m=1}^{\infty} \mathcal{F}\left(\nu, \frac{n}{n+1} \nu, \frac{1}{m}\right) .
$$

Indeed, if $\varphi \in \mathcal{F}\left(\nu, \frac{n}{n+1} \nu, \frac{1}{m}\right)$, then there exists $E \in \Sigma$ with

$$
\nu(E) \geq \frac{1}{m} \quad \text { and } \quad|\varphi|(E) \leq \frac{n}{n+1} \nu(E) .
$$

Hence $|\varphi|(E)<\nu(E)$, and so $\varphi \notin \mathcal{A}_{\nu}(X)$.

Conversely, let $\varphi \in \operatorname{ca}(\Sigma, X) \backslash \mathcal{A}_{\nu}(X)$, and fix $E_{0} \in \Sigma$ with $|\varphi|\left(E_{0}\right)<$ $\nu\left(E_{0}\right)$. Choose $n_{0}$ so that

$$
|\varphi|\left(E_{0}\right)<\frac{n_{0}}{n_{0}+1} \nu\left(E_{0}\right) .
$$

According to the Hahn decomposition theorem, applied to the measure

$$
\frac{n_{0}}{n_{0}+1} \nu-|\varphi| \quad \text { on }\left\{F \in \Sigma: F \subset E_{0}\right\}
$$

there exists $E \in \Sigma$ such that $E \subset E_{0}$,

$$
|\varphi|(F) \leq \frac{n_{0}}{n_{0}+1} \nu(F) \quad \text { whenever } F \in \Sigma \text { and } F \subset E,
$$

and $\nu(E)>0$. Thus, $\varphi \in \mathcal{F}\left(\nu, \frac{n_{0}}{n_{0}+1} \nu, \frac{1}{m}\right)$ for some $m$.

In view of (6) and Lemma 7, (b) holds for finite $\nu$.

Consider the general case and assume $\mathcal{A}_{\nu}(X)$ is nonempty. By (BDS) and $[4,(3.16 .5)]$, there exist $\nu_{1}, \nu_{2}, \ldots$ in $\mathrm{ca}_{+}(\Sigma)$ with

$$
\nu(E)=\sup \left\{\nu_{n}(E): n=1,2, \ldots\right\} \quad \text { for all } E \in \Sigma \text {. }
$$

We then have

$$
\mathcal{A}_{\nu}(X)=\bigcap_{n=1}^{\infty} \mathcal{A}_{\nu_{n}}(X),
$$

and so the general case follows from the already established special case.

5. $F_{\sigma}$-sets. To establish Theorem 5 , which is the main result of this section, we shall need, in addition to some material of Section 2, Lemmas 8 and 10 below. The latter, in turn, will be established with the help of Lemma 9.

Lemma 8. If $\lambda \in \mathrm{ca}_{+}(\Sigma)$ is nonatomic and $\lambda \neq 0$, and $X$ is infinitedimensional, then $\mathcal{E}_{\infty \cdot \lambda}(X)$ is not an $F_{\sigma}$-set in $\mathrm{ca}(\Sigma, \lambda, X)$.

Proof. Otherwise, by Lemma $5, \operatorname{ca}(\Sigma, \lambda, X) \backslash \mathcal{E}_{\infty \cdot \lambda}(X)$ would be a dense $G_{\delta}$-set in $\operatorname{ca}(\Sigma, \lambda, X)$. On the other hand, $\mathcal{E}_{\infty \cdot \lambda}(X)$ is also a dense $G_{\delta}$-set in $\mathrm{ca}(\Sigma, \lambda, X)$, by [3, Theorem 4.1] (see also Theorem 2 , (iv) $\Rightarrow(\mathrm{i})$, and Theorem 4 (a) above). Thus, we get a contradiction with the Baire category theorem. 
Given $\lambda \in \mathrm{ca}_{+}(\Sigma)$, we denote by $\mathrm{ca}_{\sigma}(\Sigma, \lambda, X)$ the subspace of $\operatorname{ca}(\Sigma, \lambda, X)$ consisting of all measures which are of $\sigma$-finite variation. For the purpose of the next two lemmas we denote by $\tau$ the $F$-space (i.e., complete metrizable linear) topology on $\mathrm{ca}_{\sigma}(\Sigma, \lambda, X)$ introduced in [5]. A base of neighbourhoods of zero for $\tau$ consists of the sets $\mathcal{U}_{\varepsilon}, \varepsilon>0$, where $\varphi \in \operatorname{ca}_{\sigma}(\Sigma, \lambda, X)$ is in $\mathcal{U}_{\varepsilon}$ if and only if $\|\varphi\| \leq \varepsilon$ and there exists $E \in \Sigma$ with

$$
\lambda(E) \leq \varepsilon \quad \text { and } \quad|\varphi|(S \backslash E) \leq \varepsilon .
$$

Lemma 9. For every $\lambda \in \mathrm{ca}_{+}(\Sigma)$ the set $\mathcal{E}_{\lambda}(X)$ is $\tau$-closed in $\mathrm{ca}_{\sigma}(\Sigma, \lambda, X)$.

Proof. Let $\varphi_{n} \in \mathcal{E}_{\lambda}(X)$, let $\varphi \in \operatorname{ca}_{\sigma}(\Sigma, \lambda, X)$, and let $\varphi_{n} \rightarrow \varphi$ with respect to $\tau$. We have to show that $\varphi \in \mathcal{E}_{\lambda}(X)$. In view of Lemma 1 we have $|\varphi| \leq \lambda$. To prove the opposite inequality, fix $\varepsilon>0$ and choose $n_{0}$ with $\varphi_{n_{0}}-\varphi \in \mathcal{U}_{\varepsilon}$. Then, in particular, there exists $E \in \Sigma$ with

$$
\lambda(E) \leq \varepsilon \quad \text { and } \quad\left|\varphi_{n_{0}}-\varphi\right|(S \backslash E) \leq \varepsilon .
$$

This implies, for every $F \in \Sigma$,

$$
\left|\varphi_{n_{0}}\right|(F \backslash E) \leq|\varphi|(F \backslash E)+\left|\varphi_{n_{0}}-\varphi\right|(F \backslash E) \leq|\varphi|(F)+\varepsilon .
$$

It follows that

$$
\lambda(F) \leq \lambda(F \backslash E)+\varepsilon \leq|\varphi|(F)+2 \varepsilon .
$$

Hence $\lambda(F) \leq|\varphi|(F)$, completing the proof.

The main idea of the proof of the next lemma is taken from the proof of [3, Theorem 5.2].

Lemma 10. If $\lambda \in \mathrm{ca}_{+}(\Sigma)$ is nonatomic and $\lambda \neq 0$, and $X$ is infinitedimensional, then $\mathcal{E}_{\lambda}(X)$ is not an $F_{\sigma}$-set in $\operatorname{ca}(\Sigma, \lambda, X)$.

Proof. Denote by $\tau_{0}$ the restriction of $\tau$ to $\mathcal{E}_{\lambda}(X)$. In view of Lemma 9, $\left(\mathcal{E}_{\lambda}(X), \tau_{0}\right)$ is a complete metrizable space. We claim that every $\mathcal{G} \subset \mathcal{E}_{\lambda}(X)$ with nonempty $\tau_{0}$-interior is nonclosed in ca $(\Sigma, \lambda, X)$. By the Baire category theorem, this yields the assertion.

To prove the claim, fix $\varphi \in \mathcal{G}$ and $\varepsilon>0$ such that

(7) for each $\psi \in \mathcal{E}_{\lambda}(X)$ with $\psi-\varphi \in \mathcal{U}_{\varepsilon}$ we have $\psi \in \mathcal{G}$.

Choose $E \in \Sigma$ with $0<\lambda(E)<\varepsilon$. Applying [3, Proposition 2.2], we can find, for $n=1,2, \ldots$,

$$
\omega_{n} \in \operatorname{ca}(\Sigma, X) \text { with }\left\|\omega_{n}\right\| \leq \min \left(\frac{1}{n}, \varepsilon-\left\|\varphi_{E}\right\|\right) \text { and }\left|\omega_{n}\right|=\lambda_{E} .
$$

Set $\psi_{n}=\omega_{n}+\varphi_{S \backslash E}$. It follows from (8) that $\psi_{n} \in \mathcal{E}_{\lambda}(X)$. Since $\psi_{n}-\varphi=$ $\omega_{n}-\varphi_{E}$, (8) also implies that

$$
\left\|\psi_{n}-\varphi\right\| \leq \varepsilon \quad \text { and } \quad\left|\psi_{n}-\varphi\right|(S \backslash E)=0 .
$$


In view of (7), we conclude that $\psi_{n} \in \mathcal{G}$. Set $\psi_{0}=\varphi_{S \backslash E}$. Clearly, $\psi_{0} \in$ $\operatorname{ca}(\Sigma, \lambda, X) \backslash \mathcal{E}_{\lambda}(X)$. Moreover, (8) shows that $\left\|\psi_{n}-\psi_{0}\right\| \rightarrow 0$. Thus, the claim is proved.

THEOREM 5. The following six conditions are equivalent:

(i) $\mathcal{E}_{\nu}(X)$ is an $F_{\sigma}$-set in $\mathrm{ca}(\Sigma, \nu, X)$;

(ii) $\mathcal{E}_{\nu}(X)$ is closed in $\mathrm{ca}(\Sigma, \nu, X)$;

(iii) $\mathcal{E}_{\nu}(X)$ is empty or $\nu$ is atomic or $X$ is finite-dimensional;

(iv) $\mathcal{A}_{\nu}(X)$ is an $F_{\sigma}$-set in $\mathrm{ca}(\Sigma, X)$;

(v) $\mathcal{A}_{\nu}(X)$ is closed in $\mathrm{ca}(\Sigma, X)$;

(vi) $\mathcal{A}_{\nu}(X)$ is empty or $\nu$ is atomic or $X$ is finite-dimensional.

Proof. The implications (ii) $\Rightarrow(\mathrm{i})$ and (v) $\Rightarrow$ (iv) are obvious. The implications (iii) $\Rightarrow$ (ii) and (vi) $\Rightarrow(\mathrm{v})$ are the contents of Lemmas $4(\mathrm{c})$ and 6 . The implication (iv) $\Rightarrow$ (i) follows from Lemma 1. In view of Theorem 1, (iii) implies (vi).

To complete the proof, we shall assume that (iii) does not hold and show that (i) does not then hold either. The negation of (iii) and (BDS) yield $\lambda \in \mathrm{ca}_{+}(\Sigma)$ with $\nu \equiv \lambda$ and $P \in \Sigma$ with $\lambda(P)>0$ and $\lambda_{P}$ nonatomic. Consequently, there exists $Q \in \Sigma$ such that

$$
Q \subset P \text { and } 0<\nu(Q)<\infty \text { or } \nu_{Q}(\Sigma)=\{0, \infty\} .
$$

Applying Lemma 10 in the former case and Lemma 8 in the latter, we deduce that $\mathcal{E}_{\nu_{Q}}(X)$ is not an $F_{\sigma}$-set in $\operatorname{ca}\left(\Sigma, \nu_{Q}, X\right)$. Set $R=S \backslash Q$ and

$$
\mathcal{Q}=\operatorname{ca}\left(\Sigma, \nu_{Q}, X\right), \quad \mathcal{R}=\operatorname{ca}\left(\Sigma, \nu_{R}, X\right) .
$$

Clearly, $\mathcal{Q}$ and $\mathcal{R}$ are closed subspaces of ca $(\Sigma, \nu, X)$ and the map

$$
U: \operatorname{ca}(\Sigma, \nu, X) \rightarrow \mathcal{Q} \times \mathcal{R}, \quad U(\varphi)=\left(\varphi_{Q}, \varphi_{R}\right),
$$

is a (linear) homeomorphism. Moreover, we have

$$
U\left(\mathcal{E}_{\nu}(X)\right)=\mathcal{E}_{\nu_{Q}}(X) \times \mathcal{E}_{\nu_{R}}(X) .
$$

Since $\mathcal{E}_{\nu_{Q}}(X)$ is not an $F_{\sigma}$-set in $\mathcal{Q}$ and $\mathcal{E}_{\nu_{R}}(X)$ is nonempty, the negation of (i) follows.

Corollary 1. (a) $\mathcal{E}_{\nu}(X)$ is open in $\mathrm{ca}(\Sigma, \nu, X)$ if and only if $\mathcal{E}_{\nu}(X)$ is empty or $\nu=0$.

(b) $\mathcal{A}_{\nu}(X)$ is open in $\mathrm{ca}(\Sigma, X)$ if and only if $\mathcal{A}_{\nu}(X)$ is empty or $\nu=0$.

Proof. Part (a) follows from Lemma 5. Part (b) follows from Theorem 5, (iv) $\Rightarrow(\mathrm{v})$, since $\mathrm{ca}(\Sigma, X)$ is connected.

The following corollary is a consequence of Theorems 4 and 5 .

Corollary 2. (a) $\mathcal{E}_{\nu}(X)$ is either closed or a $G_{\delta^{-}}$, but not an $F_{\sigma^{-}}$, set in $\operatorname{ca}(\Sigma, \nu, X)$.

(b) $\mathcal{A}_{\nu}(X)$ is either closed or a $G_{\delta^{-}}$, but not an $F_{\sigma^{-}}$, set in $\mathrm{ca}(\Sigma, X)$. 
6. Sets of measures with relatively compact range. With the exception of Theorem 1, such measures have not been considered in this paper so far. Nevertheless, all other previous results have natural counterparts for vector measures with relatively compact range. We shall formulate only some of those counterparts. To this end, we shall use the following notation:

$$
\begin{aligned}
c \mathcal{E}_{\nu}(X) & =\mathcal{E}_{\nu}(X) \cap \operatorname{cca}(\Sigma, X), \\
c \mathcal{A}_{\nu}(X) & =\mathcal{A}_{\nu}(X) \cap \operatorname{cca}(\Sigma, X), \\
c \mathcal{A}_{\nu}(\nu, X) & =c \mathcal{A}_{\nu}(X) \cap \operatorname{ca}(\Sigma, \nu, X), \\
\operatorname{cca}(\Sigma, \nu, X) & =\operatorname{ca}(\Sigma, \nu, X) \cap \operatorname{cca}(\Sigma, X) .
\end{aligned}
$$

THEOREM $2^{\prime}$. The following four conditions are equivalent:

(i) $c \mathcal{E}_{\nu}(X)$ is dense in cca $(\Sigma, \nu, X)$;

(ii) $c \mathcal{E}_{\nu}(X)$ is not nowhere dense in cca $(\Sigma, \nu, X)$;

(iii) $c \mathcal{E}_{\nu}(X)$ is of second category in $\mathrm{cca}(\Sigma, \nu, X)$;

(iv) either $\nu=0$, or $\nu=\infty \cdot \lambda$ for some nonatomic $\lambda \in \mathrm{ca}_{+}(\Sigma)$ and $X$ is infinite-dimensional.

(Note that Theorem $2^{\prime}$, (iv) $\Rightarrow$ (i), is due essentially to Anantharaman and Garg [1, Theorem 2.4]; see also [3, Theorem 4.1]).

TheOREM $3^{\prime}$. The following three conditions are equivalent:

(i) $c \mathcal{A}_{\nu}(X)$ is dense in cca $(\Sigma, X)$;

(ii) $c \mathcal{A}_{\nu}(\nu, X)$ is dense in cca $(\Sigma, \nu, X)$;

(iii) either $\nu=0$, or $\nu \equiv \lambda$ for some nonatomic $\lambda \in \mathrm{ca}_{+}(\Sigma)$ and $X$ is infinite-dimensional.

TheOREM 5'. The following six conditions are equivalent:

(i) $c \mathcal{E}_{\nu}(X)$ is an $F_{\sigma}$-set in $\mathrm{cca}(\Sigma, \nu, X)$;

(ii) $c \mathcal{E}_{\nu}(X)$ is closed in $\operatorname{cca}(\Sigma, \nu, X)$;

(iii) $c \mathcal{E}_{\nu}(X)$ is empty or $\nu$ is atomic or $X$ is finite-dimensional;

(iv) $c \mathcal{A}_{\nu}(X)$ is an $F_{\sigma}$-set in $\operatorname{cca}(\Sigma, X)$;

(v) $c \mathcal{A}_{\nu}(X)$ is closed in cca $(\Sigma, X)$;

(vi) $c \mathcal{A}_{\nu}(X)$ is empty or $\nu$ is atomic or $X$ is finite-dimensional.

To establish those theorems, it is enough to adapt the proofs of Theorems 2, 3 and 5, respectively. This is also true of some other "relatively compact" counterparts of our previous results (e.g., Lemma 4(a),(b)). In many cases, however, a direct application of a previous result would do (e.g., Lemma 4(c) and Theorem 4).

Finally, let us note that the adaptation of some previous arguments to the new setting involves the following obvious property of the space cca $(\Sigma, X)$ :

For all $\varphi \in \operatorname{cca}(\Sigma, X)$ and $E \in \Sigma$ we have $\varphi_{E} \in \operatorname{cca}(\Sigma, X)$. 


\section{References}

[1] R. Anantharaman and K. M. Garg, The properties of a residual set of vector measures, in: Measure Theory and its Applications (Sherbrooke, Québec, 1982), J. M. Belley, J. Dubois and P. Morales (eds.), Lecture Notes in Math. 1033, Springer, Berlin, 1983, 12-35.

[2] J. Diestel and J. J. Uhl, Jr., Vector Measures, Math. Surveys 15, Amer. Math. Soc., Providence, R.I., 1977.

[3] L. Drewnowski and Z. Lipecki, On vector measures which have everywhere infinite variation or noncompact range, Dissertationes Math. 339 (1995).

[4] J. Hoffmann-Jørgensen, Probability with a View toward Statistics, Vol. 1, Chapman \& Hall, New York and London, 1994.

[5] L. Janicka and N. J. Kalton, Vector measures of infinite variation, Bull. Acad. Polon. Sci. Sér. Sci. Math. Astronom. Phys. 25 (1977), 239-241.

[6] Z. Lipecki, Decomposition theorems for Boolean rings, with applications to semigroup-valued measures, Comment. Math. Prace Mat. 20 (1978), 397-403.

[7] —, Sequences of generous and nonatomic quasi-measures on Boolean algebras, Acta Math. Hungar. 92 (2001), 299-310.

[8] - Characteristic properties of the variation of a vector measure, Acta Sci. Math. (Szeged) 69 (2003), 57-66.

[9] K. Musiał, Absolute continuity and the range of group valued measure, Bull. Acad. Polon. Sci. Sér. Sci. Math. Astronom. Phys. 21 (1973), 105-113.

[10] N. Randrianantoanina and E. Saab, Weak compactness in the space of vector-valued measures of bounded variation, Rocky Mountain J. Math. 24 (1994), 681-688.

[11] E. Thomas, The Lebesgue-Nikodym theorem for vector valued Radon measures, Mem. Amer. Math. Soc. 139 (1974).

Institute of Mathematics

Polish Academy of Sciences

Wrocław Branch

Kopernika 18

51-617 Wrocław, Poland

E-mail: lipecki@impan.pan.wroc.pl

Received April 4, 2003

Revised version April 16, 2004 